\title{
De novo engineering of Corynebacterium glutamicum for L-proline production
}

Jiao Zhang ${ }^{1,2 \#}$, Fenghui Qian ${ }^{3 \#}$, Feng Dong ${ }^{3}$, Qingzhuo Wang ${ }^{1,2}$, Junjie Yang ${ }^{1}$, Yu Jiang ${ }^{3,4 *}$, Sheng Yang ${ }^{1,3 *}$

${ }^{1}$ Key Laboratory of Synthetic Biology, CAS Center for Excellence in Molecular Plant Sciences, Chinese Academy of Sciences, Shanghai 200032, China.

${ }^{2}$ University of Chinese Academy of Sciences, Beijing 100049, China.

${ }^{3}$ Huzhou Center of Industrial Biotechnology, Shanghai Institutes for Biological Sciences, Huzhou 313000, China.

${ }^{4}$ Shanghai Taoyusheng Biotechnology Co., Ltd, Shanghai 201203, China.

${ }^{\#}$ These authors contributed equally to this work.

* Corresponding author E-mail:

Yu Jiang: yjiang@cibt.ac.cn

Sheng Yang: syang@sibs.ac.cn 


\section{Supplementary materials}

Figure S1 Selection of different CEPs in C. glutamicum.

Figure S2 Point mutation, gene deletion, and insertion efficiency of 7 CEPs assisted the CRISPR system.

Figure S3 PCR validation of colonies of gene deletion and insertion experiments obtained by the traditional SacB system, CRISPR-FnCpf1 system, or SacB-assisted CRISPR- $F n C p f 1$ system.

Figure S4 PCR validation of colonies of gene deletion and insertion experiments obtained by the SacB-assisted CRISPR-FnCpf1 or SacB-assisted CRISPR-BbCpf1 system.

Figure S5 Colonies of gene deletion and insertion on BHISG-Kan obtained by the SacB-assisted CRISPR-FnCpf1 or SacB-assisted CRISPR-BbCpf1 system.

Table S1 Overview of transformation and gene editing efficiency via the CRISPR-Cas system in $C$. glutamicum.

Table S2 Overview of transformation and gene editing efficiency via the CRISPR-FnCpf1 and CRISPR-BbCpf1 system in C. glutamicum.

Table S3 Mutation efficiency of putA, gnd, and $g d h$ in ZQJY-1(pK18mobsacB-spec-peftu-gdh).

Table S4 Mutation efficiency of putA, gnd, and gdh in ZQJY-1.

Table S5 31 oligonucleotides for RBS replacement.

Table S6 Sequence of special RBS mutation type.

Table S7 L-proline fermentation of randomly picked colonies from the RBS engineering library.

Table S8 Fermentation of L-proline overproducing strains.

Table S9 Strains and Plasmids used in this study.

Table S10 Primers used in this study to construct plasmids. 
Table S11 Plasmids used as the template for CEP amplification. 


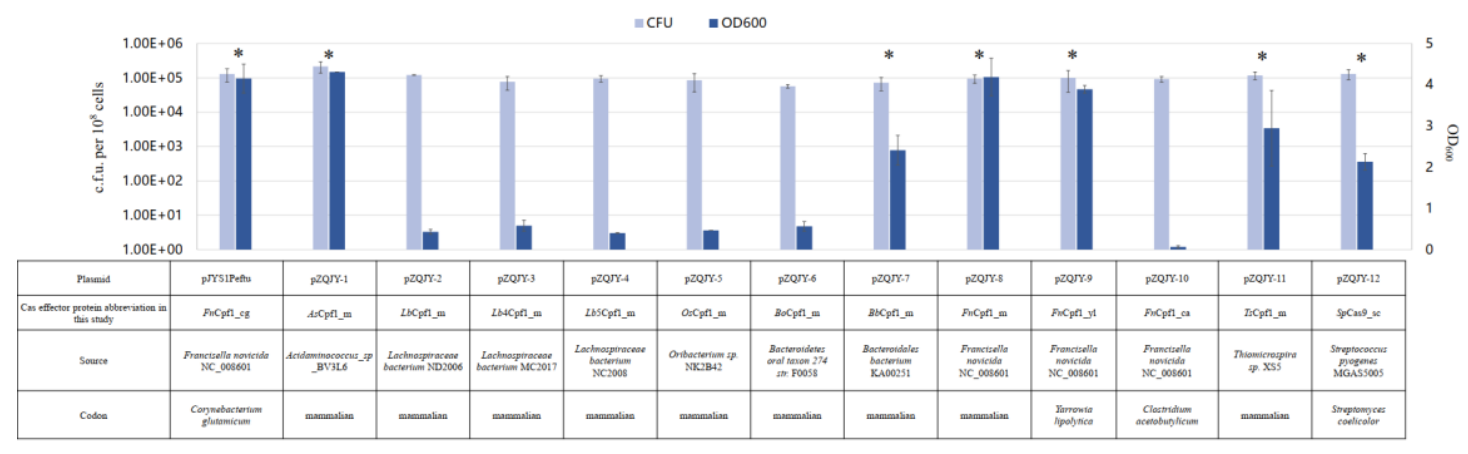

Figure S1 Selection of different CEPs in C. glutamicum. The growth of C. glutamicum transformed with pZQJY

series plasmids containing different CEPs (Thirteen CEPs from eight species (Francisella novicida NC_008601, Acidaminococcus_sp_BV3L6, Lachnospiraceae bacterium, Oribacterium sp. NK2B42, Bacteroidetes oral taxon 274 str. F0058, Bacteroidales bacterium KA00251, Thiomicrospira sp. XS5, Streptococcus pyogenes MGAS5005) corresponding to five different codon optimizations (Corynebacterium glutamicum, Yarrowia lipolytica, Clostridium acetobutylicum, Streptomyces coelicolor, mammalian)). Asterisks indicate transformations could be cultured in liquid. Experiments were conducted in duplicate. Error bars indicate \pm s.d. 


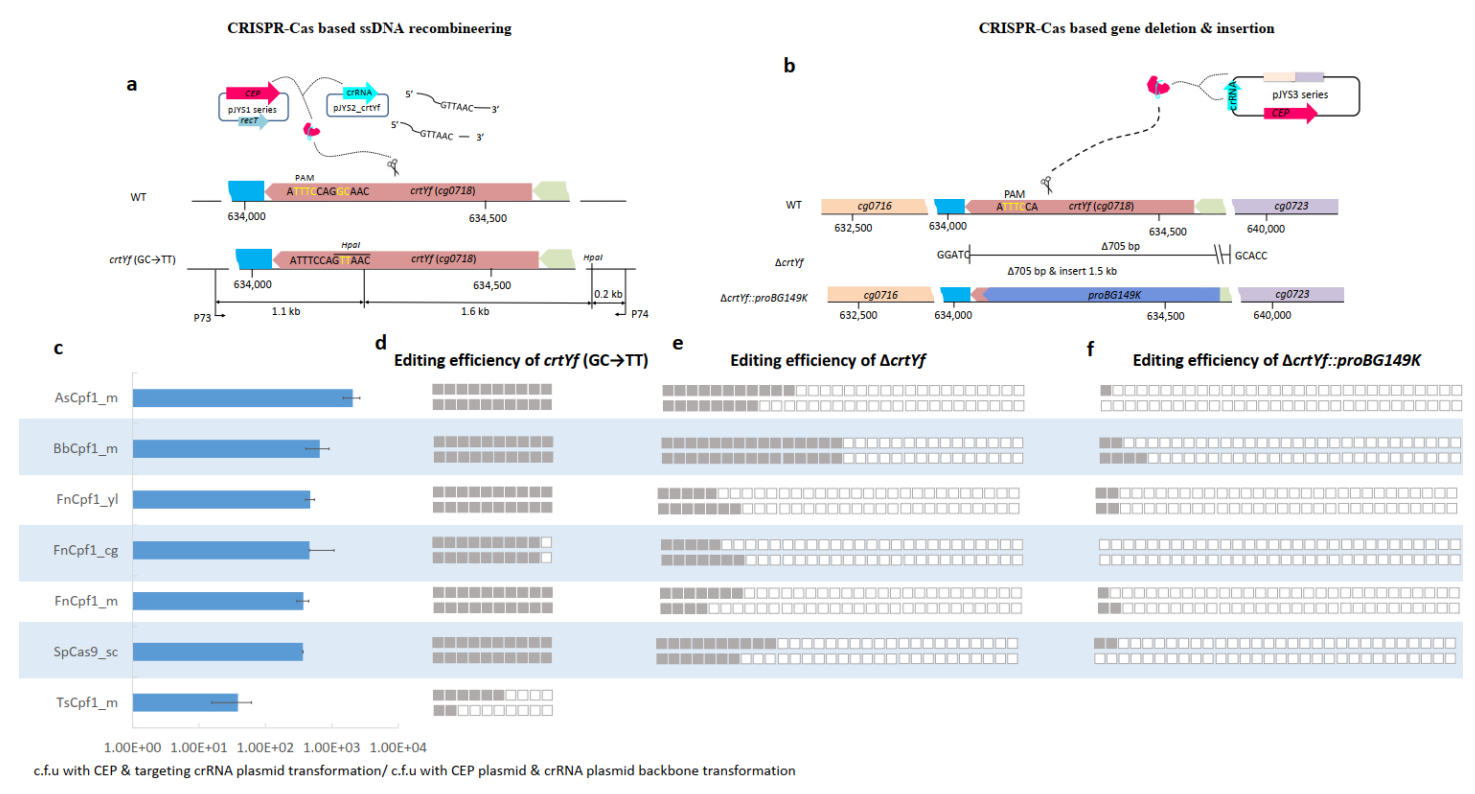

Figure S2 Point mutation, gene deletion, and insertion efficiency of 7 CEPs assisted the CRISPR system. (a)

Overview of ssDNA-mediated nucleotide substitution in $\operatorname{crt} Y f$. (b) Overview of $\operatorname{crt} Y f$ editing by the all in one

CRISPR-CEP plasmid. (c) C. glutamicum growth after transformation with the pZQJY series plasmid. (d) $c r t Y f$ mutation efficiency. (e) $c r t Y f$ deletion efficiency. (f) Gene insertion efficiency in $\operatorname{crt} Y f$ locus. Experiments were conducted in duplicate. 


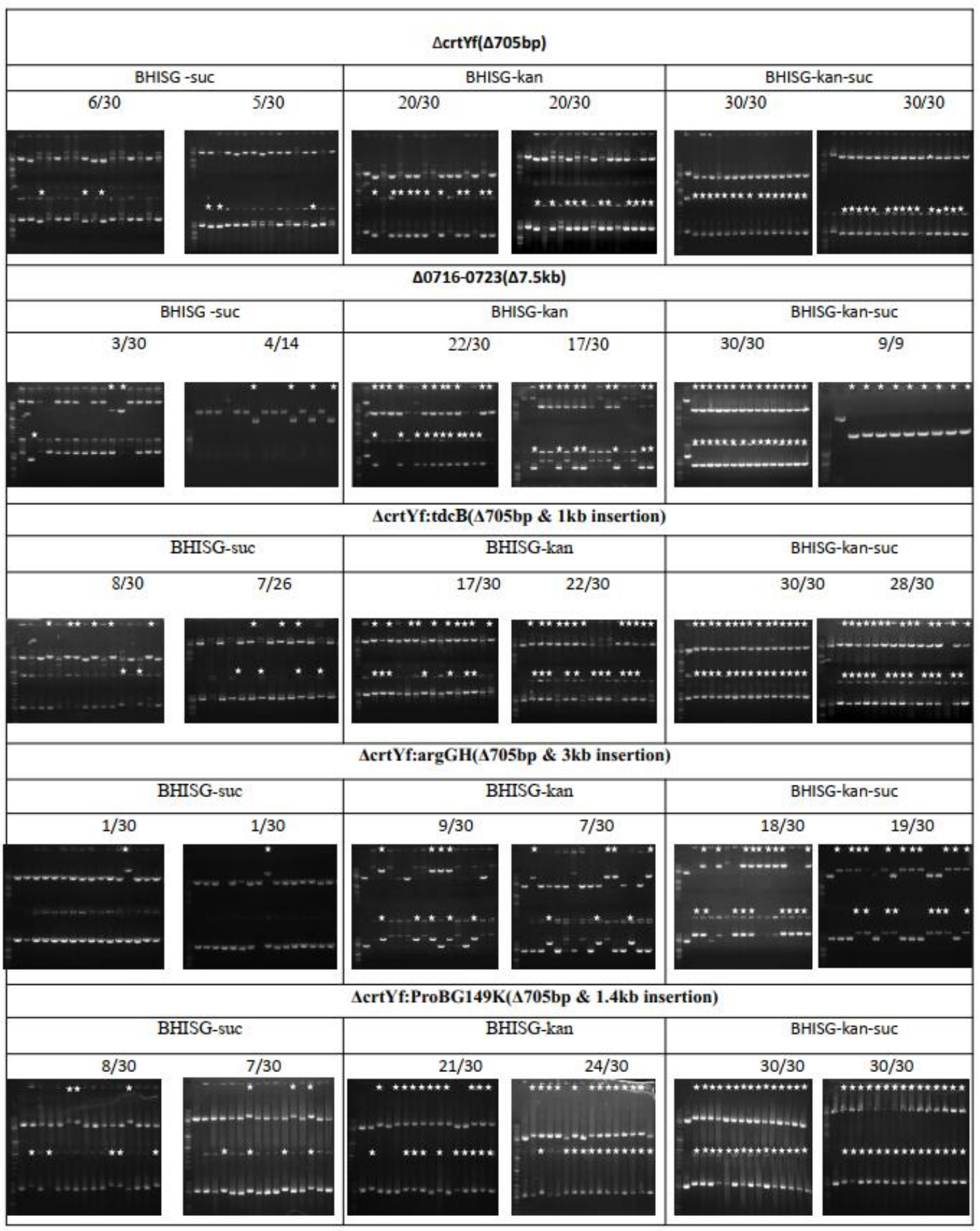

Figure S3 PCR validation of colonies of gene deletion and insertion experiments obtained by the traditional SacB system, CRISPR-FnCpfl system, or SacB-assisted CRISPR-FnCpf1 system. Experiments were conducted in duplicate. 


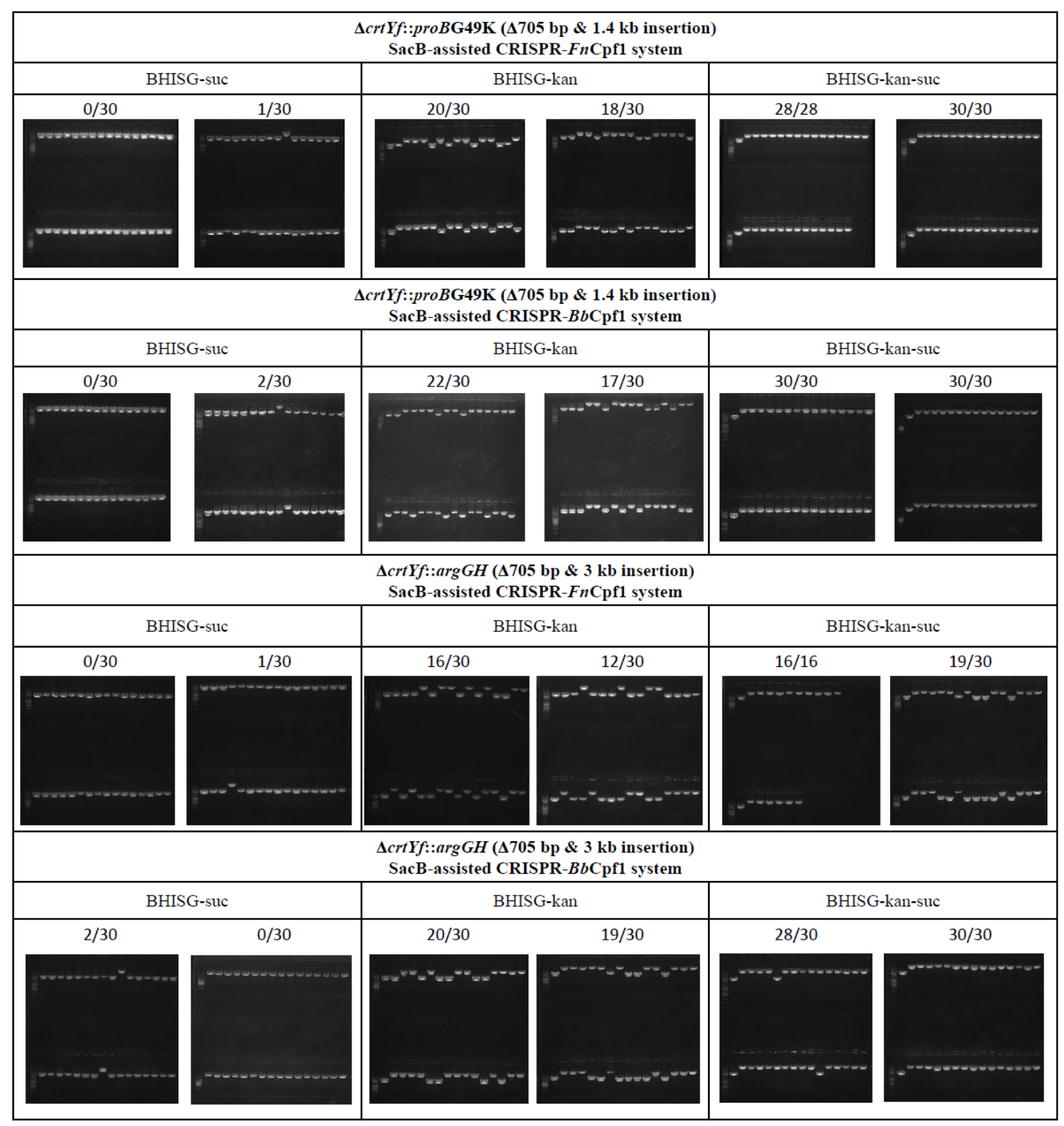

Figure S4 PCR validation of colonies of gene deletion and insertion experiments obtained by the SacB-assisted

CRISPR-FnCpf1 or SacB-assisted CRISPR-BbCpf1 system. Experiments were conducted in duplicate. 


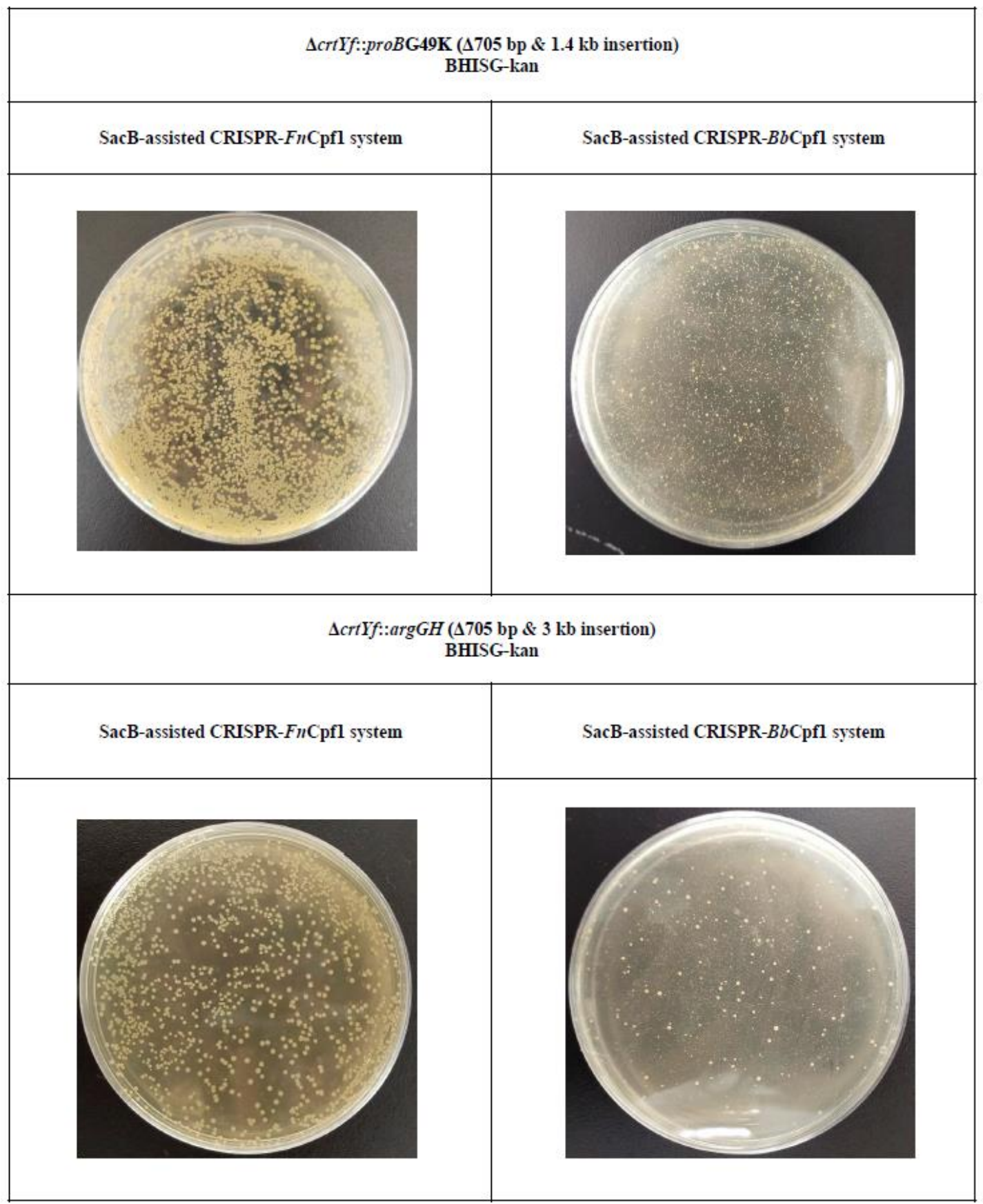

Figure S5 Colonies of gene deletion and insertion on BHISG-Kan obtained by the SacB-assisted CRISPR-FnCpf1 or SacB-assisted CRISPR-BbCpf1 system. 
Table S1 Overview of transformation and gene editing efficiency via the CRISPR-Cas system in C. glutamicum.

\begin{tabular}{|c|c|c|c|c|c|c|}
\hline Targets & Manipulation & Host & $\begin{array}{l}\text { crRNA } \\
\text { plasmid w/o } \\
\text { oligo } \\
\text { transformed }\end{array}$ & $\begin{array}{l}\text { Selection } \\
\text { plates }\end{array}$ & $\mathrm{CFU}$ & $\begin{array}{l}\text { Editing } \\
\text { efficiency }\end{array}$ \\
\hline \multirow{15}{*}{$\begin{array}{l}\operatorname{crtYf} \\
(\operatorname{cg} 0718)\end{array}$} & \multirow{3}{*}{$\begin{array}{ll}705 & \text { bp } \\
\text { deletion } & \end{array}$} & \multirow{3}{*}{$\begin{array}{l}\text { ATCC13032/ } \\
\text { pK18mobsacB } \\
\text {-spec_dcrtYf }\end{array}$} & \multirow{3}{*}{ pJYS3_crtYf } & $\begin{array}{l}\text { BHISG- } \\
\text { suc }\end{array}$ & $4.56 \pm 0.91 \times 10^{4}$ & $6 / 30,5 / 30$ \\
\hline & & & & $\begin{array}{l}\text { BHISG- } \\
\text { kan }\end{array}$ & $8.50 \pm 1.50 \times 10^{2}$ & $\begin{array}{l}20 / 30,20 / 3 \\
0\end{array}$ \\
\hline & & & & $\begin{array}{l}\text { BHISG- } \\
\text { kan-suc }\end{array}$ & $1.70 \pm 0.005 \times 10^{2}$ & $\begin{array}{l}30 / 30,30 / 3 \\
0\end{array}$ \\
\hline & \multirow{3}{*}{$7.5 \mathrm{~kb}$ deletion } & \multirow{3}{*}{$\begin{array}{l}\text { ATCC13032/ } \\
\text { pK18mobsacB } \\
\text {-spec } \\
\_\Delta 0716 / 0723\end{array}$} & \multirow{3}{*}{ pJYS3_crtYf } & $\begin{array}{l}\text { BHISG- } \\
\text { suc }\end{array}$ & $5.41 \pm 1.20 \times 10^{4}$ & $3 / 30,4 / 14$ \\
\hline & & & & $\begin{array}{l}\text { BHISG- } \\
\text { kan }\end{array}$ & $1.47 \pm 0.92 \times 10^{2}$ & $\begin{array}{l}22 / 30,17 / 3 \\
0\end{array}$ \\
\hline & & & & $\begin{array}{l}\text { BHISG- } \\
\text { kan-suc }\end{array}$ & $7.05 \pm 1.05 \times 10^{1}$ & $30 / 30,9 / 9$ \\
\hline & \multirow{3}{*}{$\begin{array}{l}705 \quad \text { bp } \\
\text { deletion \& } 1 \\
\text { kb insertion }\end{array}$} & \multirow{3}{*}{$\begin{array}{l}\text { ATCC13032/ } \\
\text { pK18mobsacB } \\
\text {-spec } \\
\text { _ } \Delta c r t Y f: t d c B\end{array}$} & \multirow{3}{*}{ pJYS3_crtYf } & $\begin{array}{l}\text { BHISG- } \\
\text { suc }\end{array}$ & $3.49 \pm 0.47 \times 10^{4}$ & $8 / 30,7 / 26$ \\
\hline & & & & $\begin{array}{l}\text { BHISG- } \\
\text { kan }\end{array}$ & $5.00 \pm 4.28 \times 10^{2}$ & $\begin{array}{l}17 / 30,22 / 3 \\
0\end{array}$ \\
\hline & & & & $\begin{array}{l}\text { BHISG- } \\
\text { kan-suc }\end{array}$ & $8.40 \pm 7.40 \times 10^{1}$ & $\begin{array}{l}30 / 30,28 / 3 \\
0\end{array}$ \\
\hline & \multirow{3}{*}{$\begin{array}{l}705 \quad \text { bp } \\
\text { deletion \& } 3 \\
\text { kb insertion }\end{array}$} & \multirow{3}{*}{$\begin{array}{l}\text { ATCC13032/ } \\
\text { pK18mobsacB } \\
\text {-spec } \\
\text { _ } \Delta c r t Y f:: \arg G \\
H\end{array}$} & \multirow{3}{*}{ pJYS3_crtYf } & $\begin{array}{l}\text { BHISG- } \\
\text { suc }\end{array}$ & $6.07 \pm 0.55 \times 10^{4}$ & $1 / 30,1 / 30$ \\
\hline & & & & $\begin{array}{l}\text { BHISG- } \\
\text { kan }\end{array}$ & $4.05 \pm 1.30 \times 10^{2}$ & $9 / 30,7 / 30$ \\
\hline & & & & $\begin{array}{l}\text { BHISG- } \\
\text { kan-suc }\end{array}$ & $3.87 \pm 0.38 \times 10^{2}$ & $\begin{array}{l}18 / 30,19 / 3 \\
0\end{array}$ \\
\hline & \multirow{3}{*}{$\begin{array}{l}705 \quad \text { bp } \\
\text { deletion \& } 1.4 \\
\text { kb insertion }\end{array}$} & \multirow{3}{*}{$\begin{array}{l}\text { ATCC13032/ } \\
\text { pK18mobsacB } \\
\text {-spec } \\
{ }_{-}{ }^{\Delta c r t Y f:: p r o B} \\
\text { G49K }\end{array}$} & \multirow{3}{*}{ pJYS3_crtYf } & $\begin{array}{l}\text { BHISG- } \\
\text { suc }\end{array}$ & $1.13 \pm 0.17 \times 10^{5}$ & $8 / 30,7 / 30$ \\
\hline & & & & $\begin{array}{l}\text { BHISG- } \\
\text { kan }\end{array}$ & $3.00 \pm 1.41 \times 10^{3}$ & $\begin{array}{l}21 / 30,24 / 3 \\
0\end{array}$ \\
\hline & & & & $\begin{array}{l}\text { BHISG- } \\
\text { kan-suc }\end{array}$ & $2.63 \pm 1.59 \times 10^{2}$ & $\begin{array}{l}30 / 30,30 / 3 \\
0\end{array}$ \\
\hline
\end{tabular}


Table S2 Overview of transformation and gene editing efficiency via the CRISPR- $F n$ Cpf1 and CRISPR- $B b C p f 1$ system in C. glutamicum.

\begin{tabular}{|c|c|c|c|c|c|c|}
\hline Targets & Manipulation & Host & $\begin{array}{l}\text { crRNA } \\
\text { plasmid w/o } \\
\text { oligo } \\
\text { transformed }\end{array}$ & $\begin{array}{l}\text { Selectio } \\
\text { n plates }\end{array}$ & $\mathrm{CFU}$ & $\begin{array}{l}\text { Editing } \\
\text { efficiency }\end{array}$ \\
\hline \multirow{12}{*}{$\begin{array}{l}\operatorname{crtYf} \\
(\operatorname{cg} 0718)\end{array}$} & \multirow{3}{*}{$\begin{array}{l}705 \quad \text { bp } \\
\text { deletion \& } 1.4 \\
\text { kb insertion }\end{array}$} & \multirow{3}{*}{$\begin{array}{l}\text { ATCC13032/ } \\
\text { pK18mobsacB- } \\
\text { spec } \\
\text { _ } \Delta c r t Y f:: p r o B G \\
49 \mathrm{~K}(\text { FnCpf } 1)\end{array}$} & \multirow{3}{*}{ pJYS3_crtYf } & $\begin{array}{l}\text { BHISG- } \\
\text { suc }\end{array}$ & $1.33 \pm 1.26 \times 10^{6}$ & $\begin{array}{l}0 / 30 \\
1 / 30\end{array}$ \\
\hline & & & & $\begin{array}{l}\text { BHISG- } \\
\text { kan }\end{array}$ & $6.71 \pm 1.32 \times 10^{3}$ & $\begin{array}{l}20 / 30 \\
18 / 30 \\
\end{array}$ \\
\hline & & & & $\begin{array}{l}\text { BHISG- } \\
\text { kan-suc }\end{array}$ & $4.00 \pm 1.69 \times 10^{1}$ & $\begin{array}{l}28 / 28 \\
30 / 30\end{array}$ \\
\hline & \multirow{3}{*}{$\begin{array}{l}705 \quad \text { bp } \\
\text { deletion \& } 1.4 \\
\text { kb insertion }\end{array}$} & \multirow{3}{*}{$\begin{array}{l}\text { АTCC13032/ } \\
\text { pK18mobsacB- } \\
\text { spec } \\
\text { _ } \Delta c r t Y f:: p r o B G \\
49 \mathrm{~K} \text { (BbCpf1) }\end{array}$} & \multirow{3}{*}{ pJYS3_crtYf } & $\begin{array}{l}\text { BHISG- } \\
\text { suc }\end{array}$ & $1.43 \pm 1.19 \times 10^{6}$ & $\begin{array}{l}0 / 30 \\
2 / 30\end{array}$ \\
\hline & & & & $\begin{array}{l}\text { BHISG- } \\
\text { kan }\end{array}$ & $1.32 \pm 0.04 \times 10^{4}$ & $\begin{array}{l}22 / 30 \\
17 / 30\end{array}$ \\
\hline & & & & $\begin{array}{l}\text { BHISG- } \\
\text { kan-suc }\end{array}$ & $3.95 \pm 0.21 \times 10^{1}$ & $\begin{array}{l}30 / 30 \\
30 / 30\end{array}$ \\
\hline & \multirow{3}{*}{$\begin{array}{l}705 \quad \text { bp } \\
\text { deletion \& } 3 \\
\text { kb insertion }\end{array}$} & \multirow{3}{*}{$\begin{array}{l}\text { АTCC13032/ } \\
\text { pK18mobsacB- } \\
\text { spec } \\
\text { _ } \Delta c r t Y f:: \arg G H \\
(\text { FnCpf1) }\end{array}$} & \multirow{3}{*}{ pJYS3_crtYf } & $\begin{array}{l}\text { BHISG- } \\
\text { suc }\end{array}$ & $2.65 \pm 2.39 \times 10^{6}$ & $\begin{array}{l}0 / 30 \\
1 / 30\end{array}$ \\
\hline & & & & $\begin{array}{l}\text { BHISG- } \\
\text { kan }\end{array}$ & $3.53 \pm 0.18 \times 10^{3}$ & $\begin{array}{l}16 / 30 \\
12 / 30\end{array}$ \\
\hline & & & & $\begin{array}{l}\text { BHISG- } \\
\text { kan-suc }\end{array}$ & $2.90 \pm 1.84 \times 10^{1}$ & $\begin{array}{l}16 / 16 \\
19 / 30\end{array}$ \\
\hline & \multirow{3}{*}{$\begin{array}{l}705 \quad b p \\
\text { deletion \& } 3 \\
\text { kb insertion }\end{array}$} & \multirow{3}{*}{$\begin{array}{l}\text { ATCC13032/ } \\
\text { pK18mobsacB- } \\
\text { spec } \\
\text { _ } \Delta c r t Y f:: \arg G H \\
\text { (BbCpf1) }\end{array}$} & \multirow{3}{*}{ pJYS3_crtYf } & $\begin{array}{l}\text { BHISG- } \\
\text { suc }\end{array}$ & $2.31 \pm 1.70 \times 10^{6}$ & $\begin{array}{l}2 / 30 \\
0 / 30\end{array}$ \\
\hline & & & & $\begin{array}{l}\text { BHISG- } \\
\text { kan }\end{array}$ & $7.13 \pm 1.79 \times 10^{3}$ & $\begin{array}{l}20 / 30 \\
19 / 30\end{array}$ \\
\hline & & & & $\begin{array}{l}\text { BHISG- } \\
\text { kan-suc }\end{array}$ & $4.30 \pm 0.57 \times 10^{1}$ & $\begin{array}{l}28 / 30 \\
30 / 30\end{array}$ \\
\hline
\end{tabular}


Table S3 Mutation efficiency of putA, gnd, and $g d h$ in ZQJY-1(pK18mobsacB-spec-peftu- $g d h$ )

\begin{tabular}{|c|c|c|c|c|c|}
\hline \multirow[b]{2}{*}{ Plasmid } & \multirow[b]{2}{*}{$\begin{array}{l}\text { Transfor } \\
\text { mation }\end{array}$} & gnd & putA & $g d h$ & \multirow[b]{2}{*}{$\mathrm{CFU}$} \\
\hline & & $\begin{array}{l}\text { TGGCTCCGACGAGA } \\
\text { ACAACTEGG ( C6- } \\
\text { T6) }\end{array}$ & $\begin{array}{l}\text { GGATCGAGTGGTT } \\
\text { CGAACTGAGG (C5- } \\
\text { T5) }\end{array}$ & $\begin{array}{l}\text { CGATTTTAA } \\
\text { AGTGTGTAC } \\
\text { CTGGG }\end{array}$ & \\
\hline \multirow{12}{*}{$\begin{array}{l}\text { pkts-ptac- } \\
\text { BE3- putA- } \\
\text { gnd- gdh }\end{array}$} & 1 & mixed (C6C7-T6T7) & mixed (C5-T5) & Insert Peftu & \multirow{12}{*}{$1.34 \times 10^{2}$} \\
\hline & 2 & mixed (C6-T6) & mixed (C5-T5) & Insert Peftu & \\
\hline & 3 & pure (C6-T6) & mixed (C5-T5) & Insert Peftu & \\
\hline & 4 & pure (C6-T6) & mixed (C5-T5) & Insert Peftu & \\
\hline & 5 & mixed (C6C7-T6T7) & mixed (C5-T5) & 0 & \\
\hline & 6 & pure (C6-T6) & mixed (C5-T5) & - & \\
\hline & 7 & pure (C6-T6) & mixed (C5-T5) & Insert Peftu & \\
\hline & 8 & pure (C6C7-T6T7) & mixed (C5-T5) & Insert Peftu & \\
\hline & 9 & pure (C6C7-T6T7) & mixed (C5-T5) & Insert Peftu & \\
\hline & 10 & pure (C6-T6) & mixed (C5-T5) & - & \\
\hline & 11 & pure (C6C7-T6T7) & mixed (C5-T5) & Insert Peftu & \\
\hline & 12 & pure (C6C7-T6T7) & mixed (C5-T5) & Insert Peftu & \\
\hline
\end{tabular}

Strain ZQJY-1 with integrated pk18mobsacB-spec-Peftu-gdh was transformed with the cytosine base editor plasmid pkts-ptac-BE3-putA-gnd-gdh containing crRNAs simultaneously targeting putA, gnd, and gdh. 12 transformants were picked to verify the genotype. "Mixed" means that the colony contains a mixed genotype of C or T in the expected editing window that needs to be further separated; "Pure" means the colony was fully edited (C to T); and "insert Peftu" means the promoter was successfully inserted before $g d h$. 
Table S4 Mutation efficiency of putA, gnd, and $g d h$ in ZQJY-1.

\begin{tabular}{|c|c|c|c|c|}
\hline \multirow{3}{*}{$\begin{array}{l}\text { Transforma } \\
\text { tion }\end{array}$} & gnd & putA & $g d h$ & Genotype \\
\hline & TGGCTCCGACGAGAA & GGATCGAGTGGTTCG & CGATTTTAAAGT & \\
\hline & CAACT $\underline{G G}$ (C6-T6) & AACTG $\underline{A G G}(\mathrm{C} 5-\mathrm{T} 5)$ & GTGTACCT $\underline{\text { GGG }}$ & \\
\hline $1-1$ & mutant (pure) (C6-T6) & mutant (pure) & Insert Peftu & gnd, putA, gdh \\
\hline $1-2$ & $\begin{array}{l}\text { mutant } \quad(\text { pure })(\mathrm{C} 6 \mathrm{C} 7- \\
\text { T6T7) }\end{array}$ & mutant (mixed) & Insert Peftu & \\
\hline $2-1$ & mutant (mixed) & - & - & \\
\hline $2-2$ & $\begin{array}{l}\text { mutant (pure) (C6C7- } \\
\text { T6T7) }\end{array}$ & mutant (pure) & Insert Peftu & gnd, putA, gdh \\
\hline $3-1$ & mutant (pure) (C6-T6) & wild type & Insert Peftu & gnd, gdh \\
\hline $3-2$ & wild type & wild type & Insert Peftu & $g d h$ \\
\hline $4-1$ & wild type & wild type & Insert Peftu & $g d h$ \\
\hline $4-2$ & mutant (pure) (C6-T6) & wild type & Insert Peftu & gnd, gdh \\
\hline $7-1$ & $\begin{array}{l}\text { mutant (pure) (C6C7- } \\
\text { T6T7) }\end{array}$ & wild type & Insert Peftu & gnd, gdh \\
\hline $7-2$ & mutant (pure) (C6-T6) & wild type & Insert peftu & gnd, gdh \\
\hline $7-3$ & mutant (mixed) & - & - & \\
\hline $7-4$ & $\begin{array}{l}\text { mutant (pure) (C6C7- } \\
\text { T6T7) }\end{array}$ & mutant (pure) & Insert Peftu & gnd, putA, gdh \\
\hline 8 & wild type & wild type & Insert Peftu & $g d h$ \\
\hline 9 & mutant (pure) (C6-T6) & mutant (pure) & Insert Peftu & gnd, putA, gdh \\
\hline 11 & mutant (pure) (C6-T6) & mutant (mixed) & Insert Peftu & \\
\hline 12 & $\begin{array}{l}\text { mutant (pure) (C6C7- } \\
\text { T6T7) }\end{array}$ & mutant (pure) & Insert Peftu & gnd, putA, gdh \\
\hline
\end{tabular}

The mutation efficiency was calculated after a single round streaking to cure pkts-ptac-BE3-gnd-putA-gdh. "Mixed"

means that the colony contains a mixed genotype of $\mathrm{C}$ or $\mathrm{T}$ in the expected editing window that needs to be further separated; "Pure" means the colony was fully edited (C to T); and "insert Peftu" means the promoter was successfully inserted before $g d h$. 
Table S5 31 oligonucleotides for RBS replacement.

\begin{tabular}{|c|c|c|}
\hline Number & Primers & Sequence 5 ' to 3 ' \\
\hline 1 & odhARBS(AgAA) & ctcaataaacctcaagaagcaaggtaaagAgAAgagtacetgccgtgagcagcgctag \\
\hline 2 & odhARBS(AgAT) & ctcaataaacctcaagaagcaaggtaaagAgATgagtacctgccgtgagcagcgctag \\
\hline 3 & odhARBS(AgAC) & ctcaataaacctcaagaagcaaggtaaagAgACgagtacctgccgtgagcagcgctag \\
\hline 4 & odhARBS(AgAG) & ctcaataaacctcaagaagcaaggtaaagAgAGgagtacetgccgtgagcagcgctag \\
\hline 5 & odhARBS(AgGA) & ctcaataaaccctcaagaagcaaggtaaagAgGAgagtacctgccgtgagcagcgctag \\
\hline 6 & odhARBS(AgGT) & ctcaataaaccctcaagaagcaaggtaaagAgGTgagtacctgccgtgagcagcgctag \\
\hline 7 & odhARBS(AgGG) & ctcaataaaccctcaagaagcaaggtaaagAgGGgagtacctgccgtgagcagcgctag \\
\hline 8 & odhARBS(TgAA) & ctcaataaacctcaagaagcaaggtaaagTgAAgagtacctgccgtgagcagcgctag \\
\hline 9 & odhARBS(TgAT) & ctcaataaaccctcaagaagcaaggtaaagTgATgagtacctgccgtgagcagcgctag \\
\hline 10 & odhARBS(TgAC) & ctcaataaacctcaagaagcaaggtaaagTgACgagtacctgecgtgagcagcgctag \\
\hline 11 & odhARBS(TgAG) & ctcaataaacctcaagaagcaaggtaaagTgAGgagtacctgccgtgagcagcgctag \\
\hline 12 & odhARBS(TgGA) & ctcaataaacctcaagaagcaaggtaaagTgGAgagtacctgccgtgagcagcgctag \\
\hline 13 & odhARBS(TgGT) & ctcaataaaccctcaagaagcaaggtaaagTgGTgagtacctgccgtgagcagcgctag \\
\hline 14 & odhARBS(TgGC) & ctcaataaacctcaagaagcaaggtaaagTgGCgagtacctgccgtgagcagcgctag \\
\hline 15 & odhARBS(TgGG) & ctcaataaacctcaagaagcaaggtaaagTgGGgagtacctgccgtgagcagcgctag \\
\hline 16 & odhARBS(CgAA) & ctcaataaacctcaagaagcaaggtaaagCgAAgagtacctgccgtgagcagcgctag \\
\hline 17 & odhARBS(CgAT) & ctcaataaaccctcaagaagcaaggtaaagCgATgagtacctgccgtgagcagcgctag \\
\hline 18 & odhARBS(CgAC) & ctcaataaacctcaagaagcaaggtaaagCgACgagtacctgccgtgagcagcgctag \\
\hline 19 & odhARBS(CgAG) & ctcaataaaccctcaagaagcaaggtaaagCgAGgagtacctgccgtgagcagcgctag \\
\hline 20 & odhARBS(CgGA) & ctcaataaaccctcaagaagcaaggtaaagCgGAgagtacctgccgtgagcagcgctag \\
\hline 21 & odhARBS(CgGT) & ctcaataaaccctcaagaagcaaggtaaagCgGTgagtacctgccgtgagcagcgctag \\
\hline 22 & odhARBS(CgGC) & ctcaataaaccctcaagaagcaaggtaaagCgGCgagtacctgccgtgagcagcgctag \\
\hline 23 & odhARBS(CgGG) & ctcaataaaccctcaagaagcaaggtaaagCgGGgagtacctgccgtgagcagcgctag \\
\hline 24 & odhARBS(GgAA) & ctcaataaaccctcaagaagcaaggtaaagGgAAgagtacctgccgtgagcagcgctag \\
\hline 25 & odhARBS(GgAT) & ctcaataaacctcaagaagcaaggtaaagGgATgagtacctgccgtgagcagcgctag \\
\hline 26 & odhARBS(GgAC) & ctcaataaaccctcaagaagcaaggtaaagGgACgagtacctgccgtgagcagcgctag \\
\hline 27 & odhARBS(GgAG) & ctcaataaacctcaagaagcaaggtaaagGgAGgagtacetgccgtgagcagcgctag \\
\hline 28 & odhARBS(GgGA) & ctcaataaaccctcaagaagcaaggtaaagGgGAgagtacctgccgtgagcagcgctag \\
\hline 29 & odhARBS(GgGT) & ctcaataaaccctcaagaagcaaggtaaagGgGTgagtacctgccgtgagcagcgctag \\
\hline 30 & odhARBS(GgGC) & ctcaataaaccctcaagaagcaaggtaaagGgGCgagtacctgccgtgagcagcgctag \\
\hline 31 & odhARBS(GgGG) & ctcaataaaccctcaagaagcaaggtaaagGgGGgagtacetgccgtgagcagcgctag \\
\hline
\end{tabular}

* The PAM regions are underlined with single lines; the substitution nucleotides are indicated in yellow. 
Table S6 Sequence of special RBS mutation type.

\begin{tabular}{|c|c|c|}
\hline Transformation & RBS & Sequence \\
\hline & WT & aataaaccctcaagaagcaaggaaaagaggcgagtacctgccgtg \\
\hline $1-02$ & RBS deficiency & aataaaccctcaagaagcaaggga \\
\hline $1-08$ & RBS deficiency & aataaaccctcaagaagcaag ag \\
\hline $1-15$ & RBS deficiency & aataaaccctcaagaagca gag \\
\hline $1-25$ & Mutation & aataaaccctcaagaaacaaggaaaagaggcgagtacctgccgtg \\
\hline $1-27$ & RBS deficiency & aataaaccctcaagaagcaag agag \\
\hline $2-05$ & RBS deficiency & aataaaccctcaagaagcaag gtag gagtacctgccgtg \\
\hline $2-25$ & Mutation & aataaacc agaagcaaggaaaagaggcgagtacctgccgtg \\
\hline $2-44$ & GAAGG & \\
\hline
\end{tabular}

* The PAM regions are underlined with single lines; the RBS are indicated in yellow. 
Table S7 L-proline fermentation of randomly picked colonies from the RBS engineering library*.

\begin{tabular}{|c|c|c|c|}
\hline Transformants & RBS & L-proline $(\mathrm{g} / \mathrm{L})$ & L-alanine $(\mathrm{g} / \mathrm{L})$ \\
\hline 1-01 (ZQJY-6) & WT & $6.74 \pm 0.16$ & $3.01 \pm 0.66$ \\
\hline 2-05 (ZQJY-7) & RBS deficiency & $12.80 \pm 0.18$ & $1.04 \pm 0.14$ \\
\hline $1-02$ & RBS deficiency & $10.79 \pm 2.32$ & $2.43 \pm 0.46$ \\
\hline $1-03$ & AGAGG & $9.38 \pm 2.38$ & $4.14 \pm 0.04$ \\
\hline $1-07$ & TGAGG & $9.23 \pm 1.89$ & $2.82 \pm 0.26$ \\
\hline $1-08$ & RBS deficiency & $10.31 \pm 3.08$ & $1.91 \pm 0.16$ \\
\hline $1-10$ & TGAGG & 7.98 & 2.76 \\
\hline $1-15$ & RBS deficiency & 8.63 & 2.85 \\
\hline $1-18$ & AGAGG & 7.97 & 3.73 \\
\hline $1-21$ & TGAGG & 7.68 & 2.94 \\
\hline $1-24$ & AGAGG & 9.26 & 4.39 \\
\hline $1-27$ & RBS deficiency & 7.66 & 4.03 \\
\hline $1-66$ & AGAGG & $8.03 \pm 2.07$ & $4.56 \pm 0.51$ \\
\hline $1-73$ & AGAGG & $9.98 \pm 0.56$ & $4.15 \pm 0.20$ \\
\hline $1-78$ & TGAGG & $11.16 \pm 2.17$ & $1.88 \pm 0.06$ \\
\hline $1-79$ & TGAGG & $11.00 \pm 2.56$ & $1.47 \pm 0.50$ \\
\hline $1-81$ & TGAGG & $8.86 \pm 1.15$ & $1.44 \pm 0.77$ \\
\hline $1-90$ & AGAGG & $7.91 \pm 2.38$ & $3.84 \pm 0.28$ \\
\hline $2-01$ & GGAGG & $8.34 \pm 3.45$ & $3.98 \pm 0.15$ \\
\hline $2-02$ & CGAGG & $6.54 \pm 0.52$ & $0.87 \pm 0.02$ \\
\hline $2-19$ & GGAGG & $10.17 \pm 1.95$ & $3.56 \pm 0.22$ \\
\hline $2-21$ & GGAGG & $6.30 \pm 0.34$ & $3.75 \pm 0.04$ \\
\hline $2-25$ & Mutation & $7.59 \pm 0.65$ & $2.99 \pm 0.75$ \\
\hline $2-44$ & GAAGG & $8.35 \pm 2.25$ & $3.86 \pm 0.16$ \\
\hline $1-04$ & AGAGG & 6.24 & 4.34 \\
\hline $1-12$ & AGAGG & 6.20 & 3.89 \\
\hline $1-14$ & AGAGG & 6.61 & 4.16 \\
\hline $1-16$ & AGAGG & 6.62 & 4.07 \\
\hline $1-20$ & AGAGG & 6.59 & 4.13 \\
\hline $1-25$ & Mutation & 6.68 & 3.97 \\
\hline $1-26$ & AGAGG & 6.43 & 4.61 \\
\hline $1-29$ & AGAGG & 6.66 & 4.25 \\
\hline $1-30$ & AGAGG & 6.74 & 4.31 \\
\hline $1-61$ & & 9.83 & 0.86 \\
\hline $1-62$ & & 7.30 & 3.85 \\
\hline $1-63$ & & 6.69 & 4.44 \\
\hline $1-64$ & & 7.58 & 4.20 \\
\hline $1-65$ & & 7.24 & 3.94 \\
\hline
\end{tabular}




\begin{tabular}{|c|c|c|}
\hline $1-67$ & 8.84 & 3.80 \\
\hline $1-68$ & 6.59 & 4.16 \\
\hline $1-69$ & 8.35 & 2.40 \\
\hline $1-70$ & 6.47 & 3.71 \\
\hline $1-72$ & 6.33 & 4.21 \\
\hline $1-74$ & 7.19 & 4.23 \\
\hline $1-80$ & 9.19 & 2.97 \\
\hline $1-82$ & 8.92 & 2.47 \\
\hline $1-83$ & 6.37 & 3.39 \\
\hline $1-84$ & 7.95 & 4.18 \\
\hline $1-85$ & 9.10 & 2.54 \\
\hline $1-86$ & 6.75 & 3.52 \\
\hline $1-87$ & 6.00 & 4.20 \\
\hline $1-88$ & 6.78 & 3.96 \\
\hline $1-89$ & 7.39 & 3.98 \\
\hline $2-51$ & 6.85 & 3.43 \\
\hline $2-52$ & 6.61 & 2.48 \\
\hline $2-53$ & 5.99 & 4.05 \\
\hline $2-54$ & 6.19 & 3.84 \\
\hline $2-55$ & 6.60 & 4.12 \\
\hline $2-56$ & 6.61 & 4.03 \\
\hline $2-57$ & 6.42 & 4.57 \\
\hline $2-58$ & 6.74 & 4.27 \\
\hline $2-59$ & 6.22 & 4.29 \\
\hline $2-60$ & 7.98 & 3.68 \\
\hline $2-61$ & 6.58 & 4.08 \\
\hline $2-62$ & 7.28 & 4.35 \\
\hline $2-63$ & 6.65 & 4.21 \\
\hline $2-64$ & 4.44 & 2.49 \\
\hline $2-65$ & 7.69 & 2.88 \\
\hline $2-66$ & 7.99 & 2.69 \\
\hline $2-67$ & 5.18 & 2.71 \\
\hline $2-68$ & 6.40 & 1.88 \\
\hline 2-69 & 8.65 & 2.78 \\
\hline $2-70$ & 7.66 & 3.98 \\
\hline $2-71$ & 6.67 & 3.93 \\
\hline $2-72$ & 7.87 & 0.77 \\
\hline $2-73$ & 7.29 & 3.80 \\
\hline $2-74$ & 6.68 & 4.40 \\
\hline 2-75 & 7.58 & 4.16 \\
\hline $2-76$ & 7.24 & 3.89 \\
\hline
\end{tabular}




\begin{tabular}{|c|c|c|}
\hline $2-77$ & 6.45 & 3.94 \\
\hline 2-78 & 5.86 & 3.75 \\
\hline $2-79$ & 6.58 & 4.11 \\
\hline $2-80$ & 6.36 & 2.33 \\
\hline $2-81$ & 6.45 & 3.66 \\
\hline $2-82$ & 5.92 & 3.56 \\
\hline $2-83$ & 6.31 & 4.17 \\
\hline 2-84 & 5.61 & 4.29 \\
\hline $2-85$ & 7.19 & 4.19 \\
\hline $2-86$ & 6.36 & 4.02 \\
\hline $2-87$ & 5.06 & 2.92 \\
\hline $2-88$ & 6.79 & 2.96 \\
\hline 2-89 & 6.87 & 1.85 \\
\hline 2-90 & 7.01 & 1.98 \\
\hline 2-91 & 4.61 & 2.91 \\
\hline 2-92 & 5.03 & 2.26 \\
\hline 2-93 & 4.47 & 2.40 \\
\hline 2-94 & 6.35 & 3.34 \\
\hline 2-95 & 9.59 & 1.75 \\
\hline 2-96 & 8.12 & 2.47 \\
\hline 2-97 & 6.74 & 3.47 \\
\hline 2-98 & 5.98 & 4.16 \\
\hline 2-99 & 6.77 & 3.92 \\
\hline $2-100$ & 7.39 & 3.93 \\
\hline 2-101 & 5.69 & 4.06 \\
\hline 2-102 & 4.57 & 1.70 \\
\hline $2-103$ & 4.64 & 3.85 \\
\hline 2-104 & 4.77 & 1.90 \\
\hline $2-105$ & 5.28 & 1.83 \\
\hline $2-106$ & 5.07 & 4.82 \\
\hline $2-107$ & 6.92 & 2.93 \\
\hline $2-108$ & 5.87 & 3.12 \\
\hline 2-109 & 4.86 & 3.17 \\
\hline $2-110$ & 8.61 & 2.62 \\
\hline $2-111$ & 5.69 & 3.03 \\
\hline $2-112$ & 8.13 & 4.03 \\
\hline $2-113$ & 5.94 & 4.01 \\
\hline $2-114$ & 7.57 & 2.20 \\
\hline 2-115 & 6.31 & 2.18 \\
\hline 2-116 & 7.64 & 3.11 \\
\hline $2-117$ & 8.50 & 4.13 \\
\hline
\end{tabular}




\begin{tabular}{|l|l|l|l|}
$2-118$ & & 8.07 & 2.92 \\
\hline $2-119$ & 5.52 & 3.15 \\
\hline $2-120$ & 6.34 & 3.21 \\
\hline $2-121$ & 5.80 & 3.26 \\
\hline $2-122$ & 7.48 & 2.26 \\
\hline $2-123$ & 6.35 & 3.07 \\
\hline $2-124$ & 5.74 & 2.61 \\
\hline $2-125$ & 8.10 & 2.84 \\
\hline $2-126$ & 6.63 & 3.01 \\
\hline $2-127$ & & 5.61 & 3.07 \\
\hline
\end{tabular}

* Transformants with high L-proline titers in the first fermentation experiment were analyzed for fermentation in triplicate and were subjected for RBS sequencing. 
Table S8 Fermentation of L-proline overproducing strains.

\begin{tabular}{|c|c|c|}
\hline Number & Strains & L-proline $(\mathrm{g} / \mathrm{L})$ \\
\hline & ATCC13032 & $1.35 \pm 0.95$ \\
\hline ZQJY-1 & ATCC13032 ProBG149K & $4.47 \pm 1.15$ \\
\hline ZQJY-2 & ATCC13032 ProBG149K $\Delta p u t A$ & $6.05 \pm 0.63$ \\
\hline ZQJY-3 & ATCC13032 ProBG149K $\Delta p u t A \mathrm{P}_{\text {eftu }}:: g d h$ GndS361F & $6.59 \pm 0.57$ \\
\hline ZQJY-4 & ATCC13032 ProBG149K $\Delta p u t A P_{e f t u:}: g d h$ GndS361F ZwfA243T & $7.78 \pm 1.68$ \\
\hline ZQJY-5 & 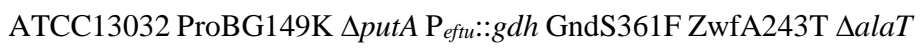 & $8.01 \pm 0.92$ \\
\hline ZQJY-6 & ATCC13032 ProBG149K $\Delta p u t A P_{e f t u:: g d h}$ GndS361F ZwfA243T $\Delta a v t A$ & $8.01 \pm 1.74$ \\
\hline ZQJY-7 & $\begin{array}{l}\text { ATCC13032 ProBG149K } \Delta p u t A P_{e f t u}:: g d h \text { GndS361F ZwfA243T } \Delta a v t A \\
\text { odhA(RBS deficiency) }\end{array}$ & $11.16 \pm 0.95$ \\
\hline ZQJY-8 & ZQJY-7/pXMJ19-ProBG149K & $11.37 \pm 1.16$ \\
\hline ZQJY-9 & ZQJY-7/pXMJ19- Peftu::ProBG149K & $18.42 \pm 0.51$ \\
\hline ZQJY-7P & ZQJY-7DcrtYf Peftu::ProBG149K & $2.14 \pm 0.04$ \\
\hline ZQJY-10 & ZQJY-7P/pXMJ19- Peftu::ProBG149K & $14.21 \pm 0.90$ \\
\hline
\end{tabular}


Table S9 Strains and Plasmids used in this study.

\begin{tabular}{|c|c|c|}
\hline Strains and plasmids & Characteristics & $\begin{array}{l}\text { Source/ } \\
\text { reference }\end{array}$ \\
\hline \multicolumn{3}{|l|}{ Strains } \\
\hline \multicolumn{3}{|l|}{$\begin{array}{ll}\text { C. } & \text { glutamicum } \\
\text { ATCC13032 } & \\
\end{array}$} \\
\hline $\begin{array}{l}\text { ATCC13032ProBG149K } \\
\text { (ZQJY-1) }\end{array}$ & ATCC13032 ProBG149K & 1 \\
\hline ZQJY-2 & ATCC13032 ProBG149K $\Delta p u t A$ & This study \\
\hline ZQJY-3 & ATCC13032 ProBG149K $\Delta p u t A \mathrm{P}_{e f t u:}: g d h$ GndS361F & This study \\
\hline ZQJY-4 & 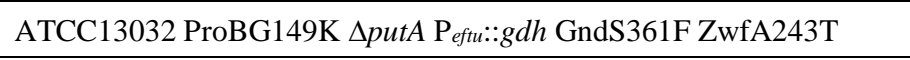 & This study \\
\hline ZQJY-5 & 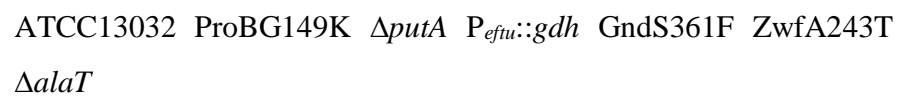 & This study \\
\hline ZQJY-6 & $\begin{array}{l}\text { ATCC13032 ProBG149K } \Delta p u t A P_{\text {eftu: }: g d h \text { GndS361F ZwfA243T }} \\
\text { savtA }\end{array}$ & This study \\
\hline ZQJY-7 & $\begin{array}{l}\text { ATCC13032 ProBG149K } \Delta p u t A \mathrm{P}_{\text {eftu }:: g d h \text { GndS361F ZwfA243T }} \\
\text { savtA odhA(RBS deficiency) }\end{array}$ & This study \\
\hline ZQJY-8 & ZQJY-7/pXMJ19-ProBG149K & This study \\
\hline ZQJY-9 & ZQJY-7/pXMJ19- Peftu::ProBG149K & This study \\
\hline ZQJY-7P & ZQJY-7AcrtYf Peftu::ProBG149K & This study \\
\hline ZQJY-10 & ZQJY-7P/pXMJ19- Peftu::ProBG149K & This study \\
\hline Pro-6 & WTproBG446AP eftu:: $\mathrm{P}_{a c n} a c n * \Delta p u t A / \mathrm{pXMJ} 19-p r o B G 446 \mathrm{~A}$ & 2 \\
\hline Pro-7 & WTProBG149KP ${ }_{e f t u}: \mathrm{P}_{a c n} a c n * \Delta p u t A / \mathrm{pXMJ} 19-\mathrm{P} e f t u::$ ProBG149K & This study \\
\hline \multicolumn{3}{|l|}{ Plasmids } \\
\hline \multicolumn{3}{|l|}{ CEP plasmids } \\
\hline pJYS1Peftu & $\begin{array}{l}\mathrm{pBL1}_{\mathrm{ts}} \text { oriV } \mathrm{C}_{\text {. glutamicum }} \mathrm{Kan}^{\mathrm{r}} \mathrm{pSC} 101 \text { ori }_{E . \text { coli }} \text { PlacM-FnCpf1, Peftu- } \\
\text { RecT }\end{array}$ & 3 \\
\hline pKCcas9dO & pSG5, $\mathrm{P}_{\text {tipA-Scocas }} 9, \mathrm{P}_{\mathrm{j} 23119-\mathrm{crRNA}}$; Amp ${ }^{\mathrm{r}}$ & $\begin{array}{l}\text { 4addgene: } \\
62552\end{array}$ \\
\hline pZQJY- CEP 1 & $\begin{array}{l}\text { Derived from pJYS1Peftu and contains AsCpf1 mammalian codon- } \\
\text { optimized; } \operatorname{Kan}^{\mathrm{r}}\end{array}$ & This study \\
\hline pZQJY- CEP 2 & $\begin{array}{l}\text { Derived from pJYS1Peftu and contains } L b C p f 1 \text { mammalian codon- } \\
\text { optimized; } \operatorname{Kan}^{\mathrm{r}}\end{array}$ & This study \\
\hline pZQJY- CEP 3 & $\begin{array}{l}\text { Derived from pJYS1Peftu and contains } L b 4 \mathrm{Cpf} 1 \text { mammalian codon- } \\
\text { optimized; } \operatorname{Kan}^{\mathrm{r}}\end{array}$ & This study \\
\hline pZQJY- CEP 4 & $\begin{array}{l}\text { Derived from pJYS1Peftu and contains } L b 5 \mathrm{Cpf} 1 \text { mammalian codon- } \\
\text { optimized; } \operatorname{Kan}^{\mathrm{r}}\end{array}$ & This study \\
\hline pZQJY- CEP 5 & $\begin{array}{l}\text { Derived from pJYS1Peftu and contains } O s \mathrm{Cpf} 1 \text { mammalian codon- } \\
\text { optimized; } \operatorname{Kan}^{\mathrm{r}}\end{array}$ & This study \\
\hline pZQJY- CEP 6 & $\begin{array}{l}\text { Derived from pJYS1Peftu and contains BoCpf1 mammalian codon- } \\
\text { optimized; } \operatorname{Kan}^{\mathrm{r}}\end{array}$ & This study \\
\hline
\end{tabular}




\begin{tabular}{|c|c|c|}
\hline pZQJY- CEP 7 & $\begin{array}{l}\text { Derived from pJYS1Peftu and contains } B b C p f 1 \text { mammalian codon- } \\
\text { optimized; } \operatorname{Kan}^{\mathrm{r}}\end{array}$ & This study \\
\hline pZQJY- CEP 8 & $\begin{array}{l}\text { Derived from pJYS1Peftu and contains FnCpf1 mammalian codon- } \\
\text { optimized; Kan }{ }^{\mathrm{r}}\end{array}$ & This study \\
\hline pZQJY- CEP 9 & $\begin{array}{l}\text { Derived from pJYS1Peftu and contains FnCpf1 Yarrowia lipolytica } \\
\text { codon-optimized; } \operatorname{Kan}^{\mathrm{r}}\end{array}$ & This study \\
\hline pZQJY- CEP 10 & $\begin{array}{l}\text { Derived from pJYS1Peftu and contains FnCpf1 Clostridium } \\
\text { acetobutylicum codon-optimized; } \operatorname{Kan}^{\mathrm{r}}\end{array}$ & This study \\
\hline pZQJY- CEP 11 & $\begin{array}{l}\text { Derived from pJYS1Peftu and contains } T s \mathrm{Cpf} 1 \text { mammalian codon- } \\
\text { optimized; Kan }{ }^{\mathrm{r}}\end{array}$ & This study \\
\hline pCgCas9_recT & $\begin{array}{l}\text { Derived from pJYS1Peftu and contains SpCas9 Streptomyces } \\
\text { coelicolor codon-optimized; } \mathrm{Kan}^{\mathrm{r}}\end{array}$ & $\begin{array}{l}\text { This study } \\
\text { (Addgene } \\
\text { ID: } \\
153337 \\
\text { Molecular } \\
\text { Cloud ID: } \\
\text { 101290) }\end{array}$ \\
\hline pJYS2_crtYf & rep ori $V_{C \text {. glutamicum }} \mathrm{Sp}^{\mathrm{r}} \mathrm{pMB} 1$ ori $V_{\text {E. coli }} \mathrm{Pj} 23119$-crRNA targeting crtYf & 3 \\
\hline pCgsgRNA_crtYf & $\begin{array}{l}\text { rep ori } V_{C .} \text { glutamicum } \mathrm{Sp}^{\mathrm{r}} \mathrm{pMB} 1 \text { ori } V_{E . \text { coli }} \mathrm{Pj} 23119 \text {-crRNA targeting } \operatorname{crt} Y \text {; } \\
\mathrm{Spc}^{\mathrm{r}}\end{array}$ & $\begin{array}{l}\text { This study } \\
\text { (Addgene } \\
\text { ID: } \\
153338 \\
\text { Molecular } \\
\text { Cloud ID: } \\
\text { 101291) }\end{array}$ \\
\hline \multicolumn{3}{|c|}{ SacB-assisted CRISPR-Cpf1plasmids } \\
\hline pJYS3_AcrtYf & $\begin{array}{l}\mathrm{pBL1}^{\text {ts }} \text { oriVC. glutamicum } \operatorname{Kan}^{\mathrm{r}} \mathrm{pSC101} \text { oriVE. coli PlacM-FnCpf1, } \\
\text { Pj23119crRNA targeting crtYf, 1-kb upstream and downstream } \\
\text { homologous arms flanking } 705 \text {-bp deletion fragment inside } c r t Y f\end{array}$ & 1 \\
\hline pJYS3_crtYf & $\begin{array}{l}\text { pBL1 }^{\text {ts }} \text { oriV } C . \text { glutamicum } \operatorname{Kan}^{\mathrm{r}} \mathrm{pSC101} \text { ori } V_{E} \text {. coli } \mathrm{PlacM}-F n \mathrm{Cpf} 1 \text {, } \\
\text { Pj23119crRNA targeting crtYf }\end{array}$ & 1 \\
\hline pK18mobsacB-spec_dcrtYf & $\begin{array}{l}\text { Derived from pK18mobsacB, } \text { spec }^{\mathrm{r}} \text {, targeting 705-bp deletion fragment } \\
\text { inside } c r t Y f\end{array}$ & \\
\hline pJYS3_AcrtYf::tdcB & $\begin{array}{l}\text { Derived from } \mathrm{pJYS} 3 \_\Delta c r t Y f \text {; Psod-tdcB }(1.3 \mathrm{~kb}) \text { inserted between the } \\
\text { 1-kb upstream and downstream homologous region flaking the 705-bp } \\
\text { deletion fragment inside } c r t Y f\end{array}$ & 1 \\
\hline $\begin{array}{l}\text { pK18mobsacB- } \\
\text { spec_AcrtYf::tdcB }\end{array}$ & $\begin{array}{l}\text { Derived from } \mathrm{pK} 18 m o b s a c B \text {, } \mathrm{spec}^{\mathrm{r}} \text {, Psod-tdcB }(1.3 \mathrm{~kb}) \text { inserted } \\
\text { between the } 1-\mathrm{kb} \text { upstream and downstream homologous region } \\
\text { flaking the } 705-\mathrm{bp} \text { deletion fragment inside } c r t Y f\end{array}$ & This study \\
\hline $\begin{array}{l}\text { pK18mobsacB-spec_s } \\
\text { crtYf::ProBG149K }\end{array}$ & $\begin{array}{l}\text { Derived from } \mathrm{pK} 18 m o b s a c B, \mathrm{spec}^{\mathrm{r}} \text {, ProBG149K }(1.4 \mathrm{~kb}) \text { inserted } \\
\text { between the } 1-\mathrm{kb} \text { upstream and downstream homologous region } \\
\text { flaking the } 705-\mathrm{bp} \text { deletion fragment inside } \operatorname{crt} Y f\end{array}$ & This study \\
\hline pK18mobsacB-spec_A & Derived from $\mathrm{pK} 18 m o b s a c B, \mathrm{spec}^{\mathrm{r}}, \arg G H(3 \mathrm{~kb})$ inserted between the & This study \\
\hline
\end{tabular}




\begin{tabular}{|c|c|c|}
\hline $\operatorname{crtYf:\operatorname {arg}GH}$ & $\begin{array}{l}\text { 1-kb upstream and downstream homologous region flaking the 705-bp } \\
\text { deletion fragment inside } \operatorname{crt} Y f\end{array}$ & \\
\hline pJYS3_Acg0716/0723 & $\begin{array}{l}\text { Derived from pJYS3_AcrtYf; 1-kb upstream and downstream } \\
\text { homologous region flanking } 7.5-\mathrm{kb} \text { deletion from } c g 0716 \text { to } c g 0723\end{array}$ & 1 \\
\hline $\begin{array}{l}\text { pK18mobsacB-spec_A } \\
\text { cg0716/0723 }\end{array}$ & $\begin{array}{l}\text { Derived from pK18mobsacB, } \mathrm{spec}^{\mathrm{r}}, 1-\mathrm{kb} \text { upstream and downstream } \\
\text { homologous region flanking } 7.5-\mathrm{kb} \text { deletion from } c g 0716 \text { to } c g 0723\end{array}$ & This study \\
\hline pJYS2_putA/crtYf & $\begin{array}{l}\text { rep ori } V_{C \text {. glutamicum }} \mathrm{Sp}^{\mathrm{r}} \mathrm{pMB} 1 \text { ori } V_{E \text {. coli }} \mathrm{Pj} 23119 \text {-crRNA targeting putA } \\
\text { and } c r t Y f\end{array}$ & This study \\
\hline $\begin{array}{l}\text { pK18mobsacB-spec_A } \\
\text { IS1207 }\end{array}$ & $\begin{array}{l}\text { Derived from } \mathrm{pK} 18 m o b s a c B, \mathrm{spec}^{\mathrm{r}} \text {, targeting 200-bp deletion fragment } \\
\text { inside IS1207 }\end{array}$ & This study \\
\hline pJYS3_IS1207-1 & $\begin{array}{l}\text { pBL1 }^{\text {ts }} \text { oriV } V_{C} \text { glutamicum } \operatorname{Kan}^{\mathrm{r}} \text { pSC101 oriV } V_{E} \text { coli PlacM-FnCpf1, } \\
\text { Pj23119crRNA targeting } I S 1207 \text {, crRNA "gcggttgcgtgaacactcatc" }\end{array}$ & This study \\
\hline pJYS3_IS1207-2 & $\begin{array}{l}\text { pBL1 }^{\text {ts }} \text { oriV } V_{C} \text { glutamicum } \operatorname{Kan}^{\mathrm{r}} \text { pSC101 oriV } V_{E} \text { coli PlacM-FnCpf1, } \\
\text { Pj23119crRNA targeting IS1207, crRNA "gggtctacaccgctagcccaggtt" }\end{array}$ & This study \\
\hline \multicolumn{3}{|c|}{ plasmids for construction of proline-producing strains } \\
\hline pCMW-BE3 & & $\begin{array}{l}{ }^{5} \text { Addgene } \\
73021\end{array}$ \\
\hline pTargetF & & $\begin{array}{l}{ }^{6} \text { Addgene } \\
62226\end{array}$ \\
\hline pKts-ptac-BE3 & $\begin{array}{l}\text { pBL1 }^{\text {ts }} \text { oriV } V_{C} \text { glutamicum } \mathrm{Kan}^{\mathrm{r}} \text { repA101 ori } V_{E} \text {. coli Ptac-APOBEC- } \\
\text { Cas9(D10A)-UGI }\end{array}$ & This study \\
\hline pKts-ptac-BE3-putA & $\begin{array}{l}\text { pBL1 }^{\text {ts }} \text { ori } V_{C} . \text { glutamicum } \mathrm{Kan}^{\mathrm{r}} \text { repA101 oriV } V_{E} \text { coli Ptac-APOBEC- } \\
\text { Cas9(D10A)-UGI Pj23119-crRNA targeting putA }\end{array}$ & This study \\
\hline $\begin{array}{l}\text { pK18mobsacB-spec- } \mathrm{P}_{\text {eftu- }} \\
\text { gdh }\end{array}$ & $\begin{array}{l}\text { Derived from pK18mobsacB, } \text { spec }^{\mathrm{r}} \text {, Peftu inserted between the 1-kb } \\
\text { upstream and downstream homologous region flaking } g d h\end{array}$ & This study \\
\hline $\begin{array}{l}\text { pkts-ptac-BE3-gnd-putA- } \\
\text { gdh }\end{array}$ & $\begin{array}{l}\text { Derived from pKts-ptac-BE3 Pj23119-crRNA targeting gnd, putA, and } \\
g d h\end{array}$ & This study \\
\hline pK18mobsacB-ZwfA243T & $\begin{array}{l}\text { pK18mobsacB with DNA fragment for introducing mutation } \\
\text { ZwfA243T }\end{array}$ & This study \\
\hline pJYS2_alaT & Derived from pJYS2_crtYf targeting alaT & This study \\
\hline pJYS2_avtA & Derived from pJYS2_crtYf targeting avtA & This study \\
\hline pJYS2_odhA & Derived from pJYS2_crtYf targeting $o d h A$ & This study \\
\hline $\begin{array}{l}\text { pXMJ19- } \\
\text { Ptac::ProBG149K }\end{array}$ & $\begin{array}{l}\text { Derived from pXMJ19 overexpressing ProBG149K under Ptac } \\
\text { promoter }\end{array}$ & This study \\
\hline pXMJ19- Peftu: $_{\text {ProBG149K }}$ & $\begin{array}{l}\text { Derived from pXMJ19 overexpressing ProBG149K under } \mathrm{P}_{e f t u} \\
\text { promoter }\end{array}$ & This study \\
\hline $\begin{array}{l}\mathrm{pK} 18 m o b s a c B \\
\mathrm{P}_{e f t u}: \mathrm{P}_{a c n} a c n^{*}\end{array}$ & $\begin{array}{l}\text { Kanr; pK18mobsacB with DNA fragment for the replacement of the } \\
\text { natural promoter of the acn gene by the promoter of eftu, mutation T1A } \\
\text { into acn gene and strong RBS sequence 'AAAGGAGGA' }\end{array}$ & This study \\
\hline pK18mobsacB-proBG446A & pK18mobsacB with DNA fragment for the replacement of proBG446A & This study \\
\hline pXMJ19-proBG446A & pXMJ19 derivative for expression of proBG446A & This study \\
\hline
\end{tabular}


Table S10 Primers used in this study to construct plasmids.

\begin{tabular}{|c|c|}
\hline Primer name & Primer sequence (5'-3') \\
\hline crRNA-F & gtcgacctgcaggcatgcaagc \\
\hline pBL-plac-R & cgttcaagtcctttccaattcc \\
\hline AscpfI-F(mammalian) & gtggtaccatgtgtggaattggaaaggacttgaacgatgacacagttcgagggctttac \\
\hline AscpfI-R(mammalian) & tctacaaactcttaagcttgcatgcctgcaggtcgacctagttcctcagctcctggatg \\
\hline LbcpfI-F(mammalian) & tgtggtaccatgtgtggaattggaaaggacttgaacgatgctgaagaacgtgggcatcg \\
\hline LbcpfI-R(mammalian) & ttctacaaactcttaagcttgcatgcctgcaggtcgactcagtgtttcacgctggtctg \\
\hline Lb4cpfI-F(mammalian) & tgtggtaccatgtgtggaattggaaaggacttgaacgatgggactgtatgacggcttcg \\
\hline Lb4cpfI-R(mammalian) & ttctacaaactcttaagcttgcatgcctgcaggtcgactcaagcaagtctgctttcctg \\
\hline Lb5cpfI-F(mammalian) & tggtaccatgtgtggaattggaaaggacttgaacgatggagaactactacgacagcctg \\
\hline Lb5cpfI-R(mammalian) & gtttctacaaactcttaagcttgcatgcctgcaggtcgactcacaggctggggatgttg \\
\hline OscpfI-F(mammalian) & gtgtggtaccatgtgtggaattggaaaggacttgaacgatggagaccgagatcctgaag \\
\hline OscpfI-R(mammalian) & gtttctacaaactcttaagcttgcatgcctgcaggtcgactcataacaggttgcactgg \\
\hline BocpfI-F(mammalian ) & tgtggtaccatgtgtggaattggaaaggacttgaacgatgaggaagttcaacgagttcg \\
\hline BocpfI-R(mammalian) & tctacaaactcttaagcttgcatgcctgcaggtcgactcagtcctccagatagggcttg \\
\hline BbcpfI-F(mammalian) & gtgtggtaccatgtgtggaattggaaaggacttgaacgatgaagaagttcaccaacctg \\
\hline BbcpfI-R(mammalian) & tctacaaactcttaagcttgcatgcctgcaggtcgactcactcgttgaagaagggcttc \\
\hline FncpfI-F(mammalian) & tgtggtaccatgtgtggaattggaaaggacttgaacgatgagcatctatcaggagttcg \\
\hline FncpfI-R(mammalian) & gtttctacaaactcttaagcttgcatgcetgcaggtcgactcaattgttgcggttctgc \\
\hline $\begin{array}{l}\text { FncpfI-F(Yarrowia } \\
\text { lipolytica) }\end{array}$ & gtgtggtaccatgtgtggaattggaaaggacttgaacgatgtccatctaccaggagttc \\
\hline $\begin{array}{l}\text { FncpfI-R(Yarrowia } \\
\text { lipolytica })\end{array}$ & ttctacaaactcttaagcttgcatgcctgcaggtcgactcaggcgtagtcgggaacatc \\
\hline $\begin{array}{l}\text { FncpfI-F(Clostridium } \\
\text { acetobutylicum })\end{array}$ & tgtggtaccatgtgtggaattggaaaggacttgaacgatgagtatatatcaagaatttg \\
\hline $\begin{array}{l}\text { FncpfI-R(Clostridium } \\
\text { acetobutylicum) }\end{array}$ & ttctacaaactcttaagcttgcatgcctgcaggtcgacttaattatttctattttgtac \\
\hline TscpfI-F(mammalian) & cgtgtggtaccatgtgtggaattggaaaggacttgaacgatgaccaagaccttcgacag \\
\hline TscpfI-R(mammalian) & gtttctacaaactcttaagcttgcatgcctgcaggtcgactcattcctggtagggettc \\
\hline ckanF & gttggctacccgtgatattg \\
\hline Rect-seq-R & tggtggcgatacggatcatac \\
\hline
\end{tabular}




\begin{tabular}{|c|c|}
\hline cas9sco-F & tgtggtaccatgtgtggaattggaaaggacttgaacgatggacaagaagtactccatcg \\
\hline cas9sco-R & gtctagattaagaaataatcttcatctaaaatatacttcagtcgccgcccagctgggag \\
\hline cas9-recT-F & agtatattttagatgaagattatttcttaatctagacaagcttaagagtttgtagaaac \\
\hline pJYS2-cas9-F1 & tatcaacttgaaaaagtggcaccgagtcggtgctttttttaagcttcatatgcggtgtg \\
\hline pJYS2-cas9-F2 & tagagctagaaatagcaagttaaaataaggctagtccgttatcaacttgaaaaagtggc \\
\hline pJYS2-cas9-F3（0718） & taggtataatggatccetgegtttaaacatatttccgttttagagctagaaatagcaag \\
\hline pJYS2-cas9-R（0718） & ctatttctagctctaaaacggaaatatgtttaaacgcagggatccattatacctaggac \\
\hline pmodR-res-R & tcatgaccaaaatcccttaacgtg \\
\hline Spec(pmodR)-F & gtggaacgaaaactcacgttaagggattttggtcatgaactaataacgtaacgtgactg \\
\hline pK18-kan-zzz-F & gcgggactctggggttcgctagag \\
\hline pK18-EcoRI-R & gaattcgtaatcatgtcatagc \\
\hline pK18-crtYf-L & atttcacacaggaaacagctatgacatgattacgaattccetatccagcagtcttcctg \\
\hline pK18-crtYf-R & gtcacgacgttgtaaaacgacggccagtgccaagcttcgtgggtggctaggcaagttac \\
\hline pK18-HindIII-F & aagcttggcactggccgtcg \\
\hline pK18-kan-qdz-R & gcgaaacgatcctcatcctgtc \\
\hline pK18-spec-F & aagatctgatcaagagacaggatgaggatcgtttcgcactaataacgtaacgtgactgg \\
\hline pK18-spec-R & aaggatcgatcctctagcgaaccccagagtccegcgatctgtacatcgatatcatatgc \\
\hline pK18-7.5kb-L & ttcacacaggaaacagctatgacatgattacgaattcgcccgcctccaagtggtgggac \\
\hline $\mathrm{pK} 18-7.5 \mathrm{~kb}-\mathrm{R}$ & tcacgacgttgtaaaacgacggccagtgccaagcttattatttttatggacccaaattc \\
\hline pK18-cg0718-1F & gctatgacatgattacgaattcagcaccgctaccattcatgggt \\
\hline pK18-cg0718-1R & actagtaggcaaccatagggcaggaatcag \\
\hline pK18-cg0718-2F & actagtggaaatatgtttaaacgcagg \\
\hline pK18-cg0718-2R & cgacggccagtgccaagcttcagaactgatcggataaaagc \\
\hline pK18-ProBG149K-F & cetgcgtttaaacatatttccactagtggecgcacgctccacg \\
\hline pK18- ProBG149K -R & ctgattcctgccetatggttgcctactagtatcccaaccgcatcaacact \\
\hline pK18-argGH-F & cagtacttctgattcctgecctatggttgcctactagtagcgeccetagttcaaggctt \\
\hline pK18-argGH-R & gacgttttttcctgcgtttaaacatatttccactagtgaattgataataaatggectgt \\
\hline Spec(pmodR)-F & gtggaacgaaaactcacgttaagggattttggtcatgaactaataacgtaacgtgactg \\
\hline pj23119-R & ggatccattatacctaggac \\
\hline crtYf-z & tgtagatcaggcaaccatagggcaggaagaattcaaataaaacgaaaggctcag \\
\hline pmodR-res-R & tcatgaccaaaatcccttaacgtg \\
\hline putA-crRNA-crtYf & tctgtactccagccaggagcgcaccgaatttctactgttgtagatcaggcaaccatagg \\
\hline p-crRNA-putA & agtcctaggtataatggatccgaatttctactgttgtagatctgtactccagccaggag \\
\hline O_putA59- & cgcatcggcattgggcacgctttccttttattagtactccagccaggagcgcaccttgt \\
\hline O_crtYf59- & agtacttctgattcctgccctatggttaactggaaatatgtttaaacgcaggaaaaac \\
\hline pj23119-F & ctcgagtctagattgacagctagctcagtcctaggtataatggatcc \\
\hline crRNA-IS1207-1F & ctactgttgtagatgcggttgcgtgaacactcatcatttaaataaaacgaaaggctcag \\
\hline crRNA-IS1207-1R & tttcgttttatttaaatgatgagtgttcacgcaaccgcatctacaacagtagaaattcg \\
\hline crRNA-IS1207-2F & ctgttgtagatgggtctacaccgctagcccaggttatttaaataaaacgaaaggctcag \\
\hline crRNA-IS1207-2R & cgttttatttaaataacctgggctagcggtgtagacccatctacaacagtagaaattcg \\
\hline pkts-F(HpaI) & tgcccgtctcactggtgaaaagaaaaaccaccctggcgttaacaggtgaacagttgttc \\
\hline pkts-R(kpnI)-1 & tctatttaaatgctagcatcgatggatcctctagaggtacctcaggtcatgattccgcg \\
\hline $\operatorname{rrnB}-\mathrm{F}(\mathrm{swaI})-1$ & ctgaggtacctctagaggatccatcgatgctagcatttaaatagagtttgtagaaacgc \\
\hline
\end{tabular}




\begin{tabular}{|c|c|}
\hline rrnB-R & gaattcagcttggctgttttgg \\
\hline UGI-F(BE3) & aaaatcttctctcatccgccaaaacagccaagctgaattcttaagaaccaccagagagc \\
\hline APOBEC1-R(BE3)-1 & cacaggaaacagaattaattaagcttaaaggaggacaactaatgagctcagagactggc \\
\hline ptac-F-1 & cattagttgtcetcctttaagcttaattaattctg \\
\hline lacIq-R & ctaacaaacaaaagtagaacaactgttcacctgttaacgccagggtggtttttcttttc \\
\hline putAC5GA-N20-F & tcctaggtataatggtaccggatcgagtggttcgaactggttttagagctagaaatagc \\
\hline spy-tem-R(swaI) & gacggatggcctttttgcgtttctacaaactctatttaaataaaaaagcaccgactcgg \\
\hline pj23119-F(kpnI) & ggggttcgcggaatcatgacctgattgacagctagctcagtcctaggtataat \\
\hline pj23119-F(kpnI)-1 & atcgecttcttgacgagttcttctgagcgggactctggggttcgcggaatcatgacetg \\
\hline pK18-HindIII-F & aagcttggcactggecgtcg \\
\hline pK18-EcoRI-R & gaattcgtaatcatgtcatagc \\
\hline gdh-L-F & atttcacacaggaaacagctatgacatgattacgaattcctggcttcggtgaggcgtcc \\
\hline gdh-L-R & aagccgctaccccacttaccetacgegectactgacactacctgggccatttagtgatg \\
\hline eftu-F & atgggaaccaccaacatcactaaatggeccaggtagtgtcagtaggcgcgtagggtaag \\
\hline eftu-R & gtcgtaatagttagagacctgctcatcaactgtcattgtatgtcctcctggacttcgtg \\
\hline gdh-R-F & aagtcgtagccaccacgaagtccaggaggacatacaatgacagttgatgagcaggtctc \\
\hline gdh-R-R & cagtcacgacgttgtaaaacgacggccagtgccaagcttgagtcttagcgttctcgccg \\
\hline pK18-F & actggecgtcgttttacaacg \\
\hline pK18-R & tcatgtcatagctgtttcctgtgtg \\
\hline zwf-aL-F & cacacaggaaacagctatgacatgattacgaattcgacaccetgtccaactccgcggc \\
\hline zwf-aL-R & cacgtccacccaagccaatatcttcagtcatggtgatctggacgtggtcaacg \\
\hline zwf-aR-F & ctacgttgaccacgtccagatcaccatgactgaagatattggettgggtggacg \\
\hline zwf-aR-R & tcacgacgttgtaaaacgacggccagtgccaagcttcgcgaggcttcattggtggactc \\
\hline alaTspc-F & tttctactgttgtagatgaggtggaatacccttgggaagttatttaaataaaacgaaag \\
\hline alaTspc-R & cgttttatttaaataacttcccaagggtattccacctcatctacaacagtagaaattcg \\
\hline alaT (TAA) 59 & actgctcgccgggccggaataatgccttattaggtggaatacccttgggaagttggaag \\
\hline avtAspc-F & tttctactgttgtagatggatacaccgaggtgattggtgatatttaaataaaacgaaag \\
\hline avtAspc-R & cgttttatttaaatatcaccaatcacctcggtgtatccatctacaacagtagaaattcg \\
\hline avtA(TAA)59 & cagagatcgctcttcgctcgggtccttaataatacaccgaggtgattggtgatcgtgag \\
\hline crRNA-odhA-TTTC-F & ctgttgtagatcttgcttcttgagggtttattgagatttaaataaaacgaaaggctcag \\
\hline crRNA-odhA-TTTC-R & cgttttatttaaatctcaataaaccctcaagaagcaagatctacaacagtagaaattcg \\
\hline $\operatorname{proB}(\operatorname{tac})-\mathrm{F}$ & aacaatttcacacaggaaacagaattaattaagcttatgcgtgagcgcatctccaacgc \\
\hline $\operatorname{proB}(\mathrm{rrnB})-\mathrm{R}$ & tcttctctcatccgccaaaacagccaagctgaattcttacgcgcggctggcgtagttgg \\
\hline Peftu(HpaI)-F & cgaaaatcctgtttgatggtggttaacgtgtcagtaggcgcgtagggtaagtggggtag \\
\hline Peftu(proB)-R & cactcgcttagcgttggagatgcgctcacgcattgtatgtcctcctggacttcgtg \\
\hline $\operatorname{proB}(\mathrm{Peftu})-\mathrm{F}$ & gtcgtagccaccacgaagtccaggaggacatacaatgcgtgagcgcatctccaacgc \\
\hline
\end{tabular}


Table S11 Plasmids used as the template for CEP amplification were synthesized by GenScript (Nanjing, China).

\begin{tabular}{|l|l|l|l|}
\hline Number & CEP & Source of strain & codon optimization \\
\hline 1 & AsCpf1 & Acidaminococcus_sp_BV3L6 & mammalian \\
\hline 2 & LbCpf1 & Lachnospiraceae bacterium ND2006 & mammalian \\
\hline 3 & Lb4Cpf1 & Lachnospiraceae bacterium MC2017 & mammalian \\
\hline 4 & Lb5Cpf1 & Lachnospiraceae bacterium NC2008 & mammalian \\
\hline 5 & OsCpf1 & Oribacterium sp. NK2B42 & mammalian \\
\hline 6 & BoCpf1 & Bacteroidetes oral taxon 274 str. F0058 & mammalian \\
\hline 7 & BbCpf1 & Bacteroidales bacterium KA00251 & mammalian \\
\hline 8 & FnCpf1 & Francisella tularensis NC_008601 & mammalian \\
\hline 9 & FnCpf1 & Francisella tularensis NC_008601 & Yarrowia lipolytica \\
\hline 10 & FnCpf1 & Francisella tularensis NC_008601 & Clostridium acetobutylicum \\
\hline 11 & $T s C p f 1$ & Thiomicrospira sp. XS5 & mammalian \\
\hline
\end{tabular}




\section{References}

[1] Jiang, Y., Qian, F., Yang, J., Liu, Y., Dong, F., Xu, C., Sun, B., Chen, B., Xu, X., Li, Y., Wang, R., and Yang, S. (2017) CRISPR-Cpf1 assisted genome editing of Corynebacterium glutamicum, Nat Commun. 8,15179 .

[2] Zhang, Y., Cai, J., Shang, X., Wang, B., Liu, S., Chai, X., Tan, T., Zhang, Y., and Wen, T. (2017) A new genome-scale metabolic model of Corynebacterium glutamicum and its application, Biotechnol Biofuels. 10, 169.

[3] Jiang, Y., Qian, F., Yang, J., Liu, Y., Dong, F., Xu, C., Sun, B., Chen, B., Xu, X., Li, Y., Wang, R., and Yang, S. (2017) CRISPR-Cpf1 assisted genome editing of Corynebacterium glutamicum, Nat Commun. 8,15179

[4] Huang, H., Zheng, G., Jiang, W., Hu, H., and Lu, Y. (2015) One-step high-efficiency CRISPR/Cas9mediated genome editing in Streptomyces, Acta Biochim Biophys Sin (Shanghai). 47, 231-243.

[5] Komor, A. C., Kim, Y. B., Packer, M. S., Zuris, J. A., and Liu, D. R. (2016) Programmable editing of a target base in genomic DNA without double-stranded DNA cleavage, Nature. 533, 420-424.

[6] Jiang, Y., Chen, B., Duan, C., Sun, B., Yang, J., and Yang, S. (2016) Multigene Editing in the Escherichia coli Genome via the CRISPR-Cas9 System (vol 81, pg 2506, 2015), Appl. Environ. Microbiol. 82, 3693-3693. 
